\title{
Dysarthria: A subject of neurology? What about endocrinology?
}

Authors: Çiğdem TURA BAHADIR, Sinan BAHADIR

Hospital: Tokat stat hospital, Tokat, TURKEY

\section{Objectives:}

Dysarthria is a motor speech disorder resulting from impairment in strength, speed and coordination of neural and/or muscular structures that are related to respiration, voice and speech. They may be classified as motor neuron dysarthria, cerebellar dysarthria and extrapyramidal dysarthria (subdivided as hypokinetic and hyperkinetic). Patients with dysarthria are evaluated primarily by neurology specialists. Though, we present our case to emphasize that dysarthria may not only result from neurological disorders but also metabolical disorders.

\section{Methods:}

A 51-year-old male patient was brought to emergency clinic with complaint of suddent speech disorder. He was awake but dysoriented. Dysarthria was present. From his background evaluation, he complaint of tingling in his hands and muscle cramps for a few years. He had a history of pathological fracture in his leg 20 years before and there was gait disturbance.

Cerebral MRI revealed T2A hyperintensity in supratentorial periventricular regions suggesting leukoaraiosis. Cranial CT scan revealed calcifications in deep gray matter, thalamus, supra- and infratentorial regions and was reported as Fahr's diseased (Figure 1).

Chvostek and Trousseau signs were positive. There was genu varum deformity in lower extremities. He had candida onychomycosis in his hands and feet and hyperpigmented regions on the anterior part of tibia secondary to convlescent exematous lesions (Figure 2). Total serum calcium, parathyroid hormone (PTH) and magnesium levels were low (Table 1). Ionized calcium study was not available.

Bone mineral density $T$ scores in the distal radius, lumbar spine and femur neck were $-5.7,-0.1$ and +1.5 respectively. The patient who had primary hypoparathyroidism and mucocutaneous candidiasis is diagnosed with polyendocrinopathy type 1. During follow-up, dysarthia was improved and cramps did nor reccur.

Figure 1: Cranial CT scan

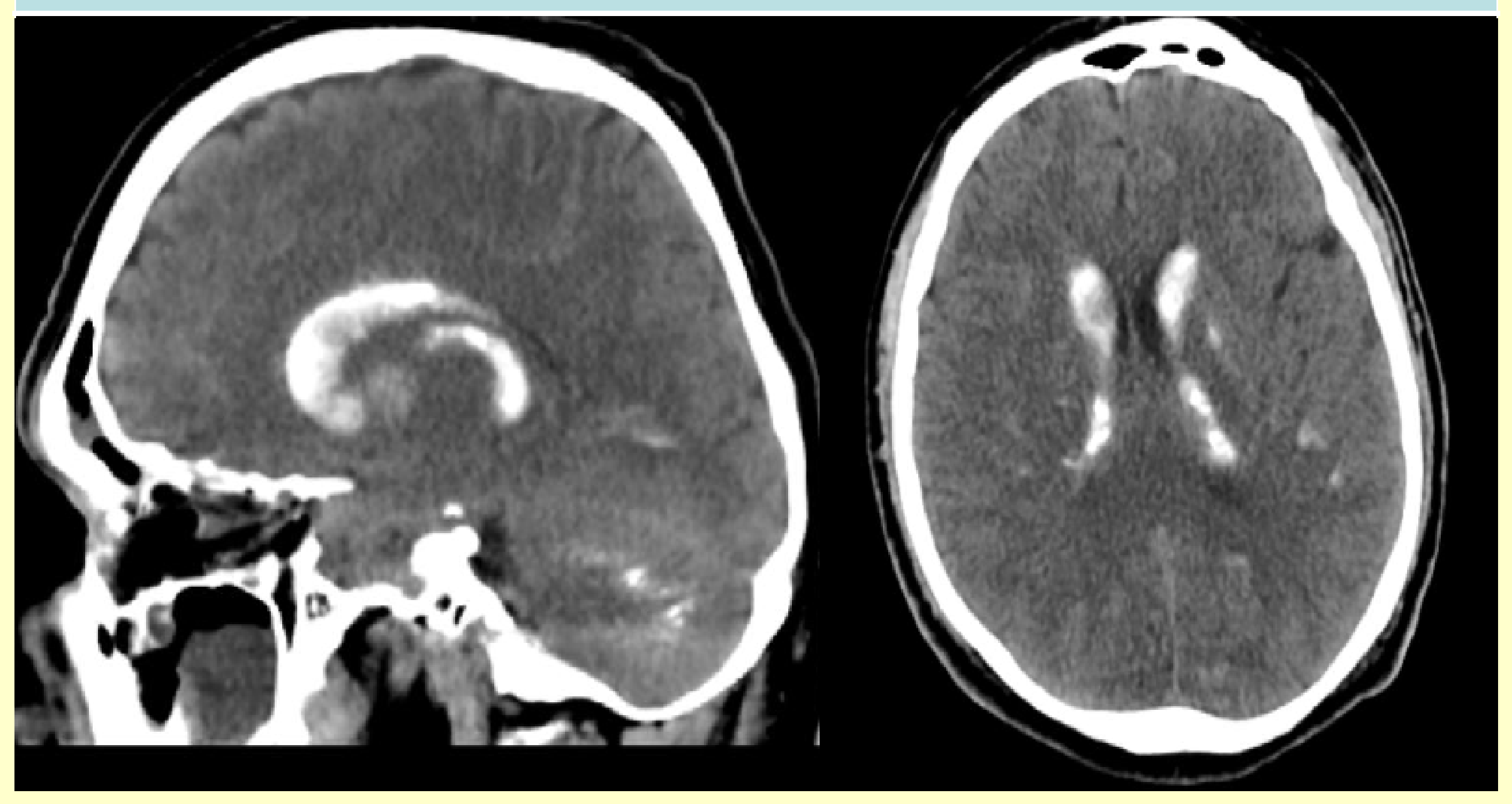

Figure 2: Patient's hands and feet following terbinafine medication

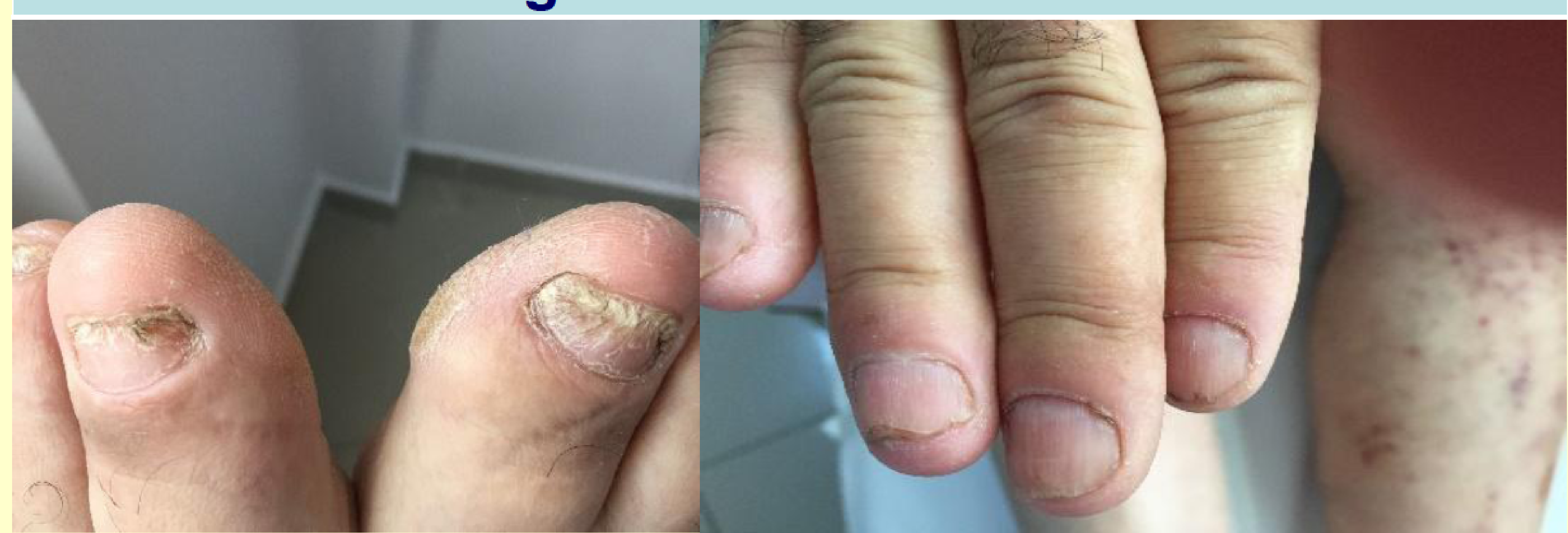

Table 1: Biochemical parameters of patient at the time of diagnosis

\begin{tabular}{l|ccc|} 
Parameter & level & & $\begin{array}{c}\text { Referance } \\
\text { range }\end{array}$ \\
\hline Total calcium & 4,1 & $\mathrm{mg} / \mathrm{dl}$ & $8,5-10$ \\
Phosphorus & 4,99 & $\mathrm{mg} / \mathrm{dl}$ & $2,5-4,5$ \\
Magnesium & 1,52 & $\mathrm{mg} / \mathrm{dl}$ & $1,6-2,6$ \\
Albumin & 3,7 & $\mathrm{~g} / \mathrm{dl}$ & $3,5-5,2$ \\
Parathormone & 1,9 & $\mathrm{pg} / \mathrm{ml}$ & $15-65$ \\
Vitamin-D & 37 & $\mathrm{ng} / \mathrm{ml}$ & $30-80$ \\
24-hr urine & 110 & $\mathrm{mmol} / \mathrm{day}$ & \\
sodium & & & \\
AlP & 436 & $\mathrm{U} / \mathrm{L}$ & $41-133$
\end{tabular}

\section{Results:}

During follow-up, dysarthia was improved and cramps did nor reccur. Celiac disease was ruled out. He was discharged with peroral calcium carbonate $3000 \mathrm{mg} /$ per day and peroral calcitriol $3 \mathrm{mcg}$ per day.

\section{Conclusions:}

Though dysarthria originates mostly from neurological disorders, metabolic disorders should also be looked for. 